rikanischen Besatzungsbehörden) und ab Oktober 1954 durch die Ford Foundation; in beiden Fällen lief dann die Finanzierung in Form von verdeckt gezahlten Geldern des amerikanischen Geheimdienstes. Möglicherweise flossen auch bereits vor Juli 1958 über den »Kongreß für kulturelle Freiheit« geheim gehaltene Gelder, der bekanntlich mit dem Monat assoziiert war und unter anderem die gesamten 1950er-Jahre zu dem Organ überaus intensive Beziehungen pflegte, sodass beispielsweise Lasky und Jaesrich regelmäßig an den Herausgebertreffen der wichtigsten »Kongreß«-Zeitschriften teilnahmen. Da aber der »Kongreß « vornehmlich von finanziellen Mitteln der CIA abhing, die anfangs direkt und später unter strengster Geheimhaltung über die »dummy foundations « - wie zum Beispiel der Ford Foundation - ausgezahlt wurden, wäre eine frühere Beteiligung der CIA beim Monat durchaus denkbar. Solange aber der genaue Zeitpunkt für die direkte oder indirekte finanzielle Beteiligung der CIA am Monat vor allem nicht durch Dokumente geklärt werden kann, kann dieser zweifelsohne wichtige Aspekt nicht endgültig entschieden werden. Fest steht nur, dass die CIA den Monat finanziell unterstützte. Dies wurde auch vom späteren Mitherausgeber Klaus Harpprecht bestätigt, der meinte, dass die geheime Finanzierung über die CIA aus haushaltstechnischen Gründen geschah, "weil die Abgeordneten und Senatoren des amerikanischen Kongresses kaum bereit gewesen wären, die Mittel für solchen Luxus $\mathrm{zu}$ genehmigen $\aleph^{263}$. Allerdings machten die Herausgeber des Monat überhaupt kein Geheimnis daraus, dass das Organ im Namen der US-amerikanischen Besatzungsbehörden herausgegeben wurde: Dies war monatlich im Impressum zu lesen und wurde von Lasky und Jaesrich im Rahmen der sogenannten Privatisierung der Zeitschrift im Septemberheft 1954 den Lesern auch ausdrücklich mitgeteilt. ${ }^{264}$

\title{
4.4 Die inhaltliche Ausrichtung des Monat unter der Ägide Laskys
}

Die inhaltliche Ausrichtung des Monat von Oktober 1948 bis April 1960 (H. I bis 139) stand ohne Zweifel in einem unmittelbaren Zusammenhang mit der speziellen Funktion der Zeitschrift in diesem Zeitraum. Hierbei lag in den ersten Jahren im Großen und Ganzen keine direkte Einflussnahme seitens der offiziellen amerikanischen Stellen vor. Nach Auffassung von Harold Hurwitz besaß Der Monat als »offizielles Organ« ungewöhnlich viel Freiheit, weil er gewisse Grenzen respektierte. ${ }^{265}$ Nach der Ablösung von staatlichen Stellen und dem Übergang 1954 zur Ford Foundation und 1958 zum »Kongreß für kulturelle Freiheit« hatte das Organ um Lasky ungewöhnlich viel Freiheit, weil er sowohl die inhaltliche Gesamtkonzeption als auch die Grenzen selbst festlegte.

Im Kern gruppierte sich die inhaltliche Ausrichtung der Zeitschrift um die beiden ideologisch aufgeladenen Themenfelder, die für die Gründung des Monat von heraus-

263 Zit. n. Martin, Orwell, Koestler und all die anderen, S. 21.

264 Siehe Der Monat 6 (1954), H. 72, Umschlagseite innen. Zudem räumte Jaesrich im Mai 1955 (Der Monat 7 [1955], H. 80, S. 180) ein, dass die Zeitschrift direkt von der Ford Foundation, einer sogenannten privaten Stiftung, subventioniert wurde. Vgl. in diesem Zusammenhang auch $\mathrm{H}$ [ellmut] ][aesrich], Das Besatzungskind, in: Der Monat 20 (1968), H. 241, S. 4; Gerd Bucerius, Liebe Leser!, in: Der Monat 21 (1969), H. 244, S. 6 f., sowie ders., Noch auf ein Wort, in: Der Monat 23 (1971), H. 270, S. 4 f.

265 Harold Hurwitz, Der heimliche Leser. Beiträge zur Soziologie des geistigen Widerstandes, Köln/Berlin 1966, S. 19. 
ragender Bedeutung waren: »Reorientation« und Antikommunismus. Bevor im Folgenden näher darauf eingegangen wird, sind vorab einige Bemerkungen angebracht, aus denen die allgemeine Linie und der generelle inhaltliche Charakter des Organs verständlich werden. Der Monat war qua definitionem eine internationale kulturpolitische Zeitschrift, in der facettenreiche Themen behandelt wurden. Neben politischen, politologischen, soziologischen, historischen oder philosophischen Themen wurden Beiträge veröffentlicht, die in den Bereich der Nationalökonomie, Literatur, Theater, Film und Kunst fielen und häufig in Form von Rezensionen erfolgte. Hierbei verstand sich die Zeitschrift ausdrücklich als ein Forum, das dazu diente, einen offenen intellektuellen Dialog zu führen. Der Charakter als offenes Diskussionsforum drückte sich auch und vor allem darin aus, dass in zahlreichen Ausgaben des Organs ein bestimmtes Thema von den Autoren unter verschiedenen Perspektiven beleuchtet wurde. Der Diskussionscharakter der Zeitschrift drückte sich zudem darin aus, dass die Redaktion unter der ständigen Rubrik »Briefe des Monats« (kurz: Leserbriefe) sowohl den Autoren als auch den Lesern die Möglichkeit einräumte, ihre Meinungen zu einzelnen Beiträgen zu veröffentlichen; nicht selten entwickelte sich aus unterschiedlichen Ansichten unter den Autoren eine inhaltliche Kontroverse. Vor dem Hintergrund der facettenreichen Themen und dem Ziel, ein breites Publikum zu erreichen, war die Redaktion grundsätzlich darum bemüht, dass die Veröffentlichungen nicht zu wissenschaftlich ausfielen. Zudem sollten die Autoren, einer angelsächsischen Tradition folgend, möglichst in einer ansprechenden, gleichwohl gut lesbaren Sprache veröffentlichen, wobei allenfalls bei soziologischen und philosophischen Beiträgen eine Ausnahme gemacht wurde. ${ }^{266}$

Im Hinblick auf das politische Selbstverständnis des Monat kann festgestellt werden, dass er sich als eine antitotalitäre Zeitschrift verstand, die, wie gesehen, den liberal-demokratischen Werten verpflichtet war. Negativ formuliert bedeutete dies folglich die Ablehnung und die Gegnerschaft zu allen nichtdemokratischen Herrschaftstypen. Damit waren zum damaligen Zeitpunkt erstens die nicht mehr existierenden Herrschaftsregime des deutschen Nationalsozialismus und des italienischen Faschismus gemeint, zweitens das zeitgenössische sowjetkommunistische Herrschaftsregime einschließlich seiner Satellitenstaaten sowie drittens die existierenden rechtsgerichteten Diktaturen in Spanien, Portugal oder auch in Mittel- und Südamerika. Als totalitäre Herrschaftssysteme wurden der Nationalsozialismus und der Sowjetkommunismus - insbesondere in seiner stalinistischen Phase - betrachtet. Vor dem Hintergrund der antitotalitären Grundposition folgte daraus auch, dass die Analyse und Beschreibung des Nationalsozialismus und des Sowjetkommunismus sowie der ausdrückliche Vergleich der beiden totalitären Regime und die zeitgenössischen Totalitarismustheorien explizit und implizit zu Schwerpunktthemen avancierten. ${ }^{267}$

Da Der Monat in der Hochphase des Kalten Krieges ins Leben gerufen wurde, gehörten infolgedessen grundsätzliche Reflexionen und politische Einschätzungen zum Ost-West-Konflikt von Anfang an zu einem wichtigen Bestandteil der Zeitschrift. Insbesondere in den ersten Jahren war dieses Thema politisch aufgeladen und besaß angesichts der Gefahr einer möglichen militärischen Konfrontation des Westens mit der Sowjetunion häufig einen existenziellen Charakter. Zu diesem Zeitpunkt kor-

266 Hochgeschwender, Freiheit in der Offensive?, S. $170 \mathrm{f}$.

267 Siehe hierzu Kap. IV. 
respondierte die Thematisierung des Ost-West-Konfliktes in zahlreichen Fällen mit genuinen Betrachtungen über das Wesen des sowjetischen Staates unter Stalin. ${ }^{268}$ In diesem Zusammenhang wurden mit Blick auf die politische Strategie des Westens in der Auseinandersetzung mit der Sowjetunion vor allem die Auffassungen von Georg F. Kennan unter den Autoren der Zeitschrift intensiv diskutiert. ${ }^{269}$ Zudem wurde in Anbetracht der Tatsache, dass die Vereinigten Staaten angesichts des Kalten Krieges Westdeutschland auch militärisch zu einem strategisch wichtigen Bündnispartner machen wollten, die Gründung der Bundeswehr und die Wiederbewaffnungsproblematik immer wieder im Monat thematisiert. ${ }^{270}$ Wobei ebendiese Betrachtungen explizit und implizit im Zusammenhang mit der speziellen Funktion der Zeitschrift standen, die Bedeutung der deutschen Westorientierung hervorzuheben, ${ }^{271}$ was wiederum mit der grundsätzlichen Thematisierung der europäischen Einigung, d.h. dem Aufbau eines politisch und ökonomisch neuen Europas, ${ }^{272}$ und dem transatlantischen Verhältnis ${ }^{273}$ koinzidierte.

268 Siehe exemplarisch Barbara Ward, Ist der Krieg mit Russland unvermeidlich?, in: Der Monat 1 (1948), H. 2, S. 3-7; James Burnham, Vom Wesen des Kommunismus, in: Ebd., S. 18-29, sowie ders., Waffen des Kalten Krieges, in: Der Monat 2 (1950), H. 20, S. 190-197.

269 Siehe hierzu George F. Kennan, Amerika und Russlands Zukunft, in: Der Monat 3 (1951), H. 34, S. 339350; »Der Westen und die Zukunft Russlands. Eine Rundfrage zu George F. Kennans Aufsatz«, in: Der Monat 4 (1951), H. 37, S. 49-67 (mit Beiträgen von Raymond Aron, Fedor Stepun, Ernst Reuter, David ]. Dallin, Max Beloff und Wilhelm Röpke), sowie: »Der Westen und die Zukunft Russlands. Zweiter Teil der Rundfrage über George Kennans Aufsatz«, in: Der Monat 4 (1951), H. 39, S. 246-260 (mit Beiträgen von Michael Karpovich, Bertrand de Jouvenel, Boris Shub und Hubert Ripka).

270 Siehe vor allem: Des Teufels Hauptwachtmeister. Eine Debatte rund um »Null-acht-Fünfzehn«, in: Der Monat 6 (1954), H. 69, S. 245-263 (mit Beiträgen von Hans Hellmut Kirst, Franz-Josef Strauß, Thilo Koch, Günther Blumentritt, Rolf Schroers, Kurt Linde, Konrad Kraske und Albert Weinstein); Walter Görlitz, Des Teufels Hauptwachtmeister, in: Der Monat 6 (1954), H. 70, S. 405-407; Heinz Karst, Von »08/15« zum Verteidigungsbeitrag. Über die zukünftige deutsche Armee, in: Der Monat 6 (1954), H. 72, S. 532-538; Fritz René Allemann, Brief aus Bonn: Die Nemesis der Ohnmacht. Wiederbewaffnung als innenpolitische Aufgabe (Teil 1), in: Der Monat 7 (1955), H. 80, S. 99-105, und Was ist eine demokratische Armee? Wiederbewaffnung als innenpolitische Aufgabe (Teil 2), in: Ebd., H. 81, S. 195201, sowie hierzu die Diskussion unter dem Titel Die deutschen Soldaten in: Ebd., H. 83, S. 415-439 (mit Beiträgen von Gerhard Ritter, Fritz Erler, Albert Kesselring, Werner Friedmann, Walter Görlitz, Hellmuth Heye, Adelbert Weinstein, Werner Picht, Bogislav v[on] Bonin und Claus ]acobi).

271 Siehe Walter Maria Guggenheimer, Die Deutschen in Europa, in: Der Monat 1 (1949), H. 4, S. 26-29; f[rançois]b[ondy], Wie westlich dürfen die Deutschen sein, in: Der Monat 3 (1951), H. 32, S. 209 f.; G[olo] M[ann], Sind die Deutschen Westeuropäer?, in: Der Monat 4 (1952), H. 42, S. 658 f.

272 Siehe bes. Bertrand de Jouvenel, Expansion und Kontraktion, in: Der Monat 1 (1949), H. 4, S. 35; Karl von Schumacher, Kann der Marshall-Plan Europa retten?, in: Ebd., S. 19-22; Wilhelm Röpke, Europäische Wirtschaftsgemeinschaft, in: Der Monat 4 (1952), H. 45, S. 227-253; Jean Fourastié, Die Wirtschaftskraft Europas, in: Der Monat 4 (1952), H. 46, S. 353-365; Richard Loewenthal [sic!], Vom Sinn und Unsinn der Wirtschaftseinheit, in: Der Monat 4 (1952), H. 47, S. 451-467; Raymond Aron, Nationale Gesundung und europäische Integration. Aufbau eines neuen Europa, in: Der Monat 5 (1953), H. 54, S. 579-592; Herbert Lüthy, Wirtschaftseinheit-Oder was sonst?, in: Der Monat 5 (1953), H. 55 , S. 26-44; Ernst Eisendrath, Europäische Wirtschaft im Wiederanstieg, in: Der Monat 5 (1953), H. 59 , S. 532-537; Pierre Emmanuel, Warum ich Europäer bin, in: Der Monat 10 (1958), H. 117, S. 26-35.

273 Siehe bes. die Diskussion unter der Überschrift Europa und Amerika. Ein transatlantisches Cespräch, in: Der Monat 5 (1952), H. 50, S. 115-194 (mit Beiträgen u. a. von Raymond Aron, Ernst Reuter, Denis W. Brogan, Melvin J. Lasky, Marion Gräfin Dönhoff); Alan Bullock, Europa und USA, in: Der Monat 
Angesichts des existierenden Stalinismus und später des Nachstalinismus schlug sich die von Lasky und seiner Redaktion vertretene Ablehnung totalitärer Regime in einer besonderen antitotalitären Antikommunismuskonzeption nieder. Obwohl das facettenreiche Totalitarismusphänomen sowohl in seiner braunen und roten Spielart intensiv thematisiert wurde, dominierte die antikommunistische Ausrichtung im Monat. Gleichwohl war die Zeitschrift in diesem Zusammenhang kein ausschließlich negativ abgrenzendes Propagandainstrument. Denn neben dem grundsätzlichen Antikommunismus - so, wie es dem gesellschaftlichen und politischen Konsens in den Vereinigten Staaten entsprach - wurden die positiv vereinnahmenden westlichen Individual- und Freiheitsrechte fokussiert, die freilich im Kontext der »Reorientation «-Funktion des Monat standen. Mit anderen Worten: Der Antikommunismus und die Hervorhebung der westlichen Werte bedingten sozusagen einander. Insofern konnte das im Monat gezeichnete Bild des sowjetkommunistischen Regimes insbesondere unter Stalin, indem die Bevölkerung in Unfreiheit lebte und der Terror des Geheimdienstapparates die gesamte Gesellschaft durchdrang, als Negativfolie für die Freiheiten in den westlichen Staaten fungieren. Vor diesem Hintergrund besaß die Auseinandersetzung mit dem komplexen Phänomen des sowjetischen Kommunismus von Anfang an einen überragenden Charakter. Zum Synonym für das totalitäre Herrschaftssystem des Stalinismus wurden im Grunde genommen die sowjetischen Konzentrationslager und infolgedessen beleuchteten zahlreiche Beiträge explizit und implizit die politische und ökonomische Bedeutung der Lager und fokussierten hierbei die Rolle des Terrors. ${ }^{274}$ Im Zusammenhang mit der intensiven Beschäftigung der sogenannten Krawtschenko- und Rousset-Prozesse im Nachkriegsfrankreich konnte dies verbunden werden mit einer scharfen Kritik an der französischen Linken und führenden Intellektuellen des Landes um Jean-Paul Sartre. ${ }^{275}$ Überhaupt war das Verhältnis der Intellektuellen zur Sowjetunion von hochrangiger Bedeutung: Einerseits wurde die Faszinationskraft, die die Sowjetunion auf zahlreiche Intellektuelle vor allem in den Ländern Ost- und Westeuropas ausübte, zur Sprache gebracht und welche Rolle hierbei die kommunistische Ideologie, sprich: der Marxismus, spielte sowie andererseits der sogenannte Desillusionierungsprozess ins Licht gerückt, der in vielen Fällen eine existenzielle Bedeutung hatte. ${ }^{276}$

5 (1953), H. 53, S. 560-563, sowie Max Frisch, Nachtrag zum »Transatlantischen Gespräch, in: Ebd., H. 59, S. 537-540.

274 Siehe vor allem Boris Shub, Lüge und Illusion, in: Der Monat 1 (1948), H. 1, S. 88-90 (Rezension); Günther Birkenfeld, Der NKWD-Staat, in: Der Monat 2 (1950), H. 18, S. 628-643; Ernest]. Salter, Gefängnis der Völker, in: Ebd., H. 24, S. 600-603 (Rezension). Die Angaben zu den Büchern erfolgen hier nach den Informationen des Monat.

275 Siehe hierzu das Kap. III mit den entsprechenden Angaben zu den im Monat veröffentlichten Beiträgen.

276 Siehe bes. die Reihe »Der Gott, der keiner war«bzw. »Ein Gott hat versagt« mit folgenden Beiträgen: Ignazio Silone, Abkehr von Moskau, in: Der Monat 2 (1950), H. 19, S. 18-39 (Teil I); Arthur Koestler, Verlorene Illusionen, in: Ebd., H. 20, S. 133-169 (Teil II); André Gide, Legende und Wirklichkeit, in: Ebd., H. 21, S. 264-281 (Teil III); Richard Wright, Der große Mythos, in: Ebd. 3 (1951), H. 25, S. 55-82 (Teil IV) ([Teil-]Abdruck des Buches Ein Gott der keiner war. Arthur Koestler, Ignazio Silone, Richard Wright, André Cide, Louis Fischer, Stephen Spender schildern ihren Weg zum Kommunismus und ihre Abkehr. Mit einem Vorwort von Richard Crossmann und einem Nachwort von Franz Borkenau, Konstanz/Zürich/Wien 1950); siehe hierzu auch speziell Bertrand Russell, Der Irrtum des Kommu- 
Überhaupt rückte die Beschreibung und Analyse des Sowjetkommunismus ausdrücklich in den Fokus des Interesses der »Lasky-Zeitschrift«. Vor diesem Hintergrund beschäftigten sich zahlreiche Veröffentlichungen mit der Genese der kommunistischen Gewaltherrschaft nach der Russischen Revolution von 1917. Angesichts der zum damaligen Zeitpunkt noch nicht vorhandenen wissenschaftlichen Forschungsliteratur war der Monat auch ein Medium, in dem internationale Kommunismusexperten, die in zahlreichen Fällen wie beispielsweise Arthur Koestler, Franz Borkenau, Richard Löwenthal oder Ruth Fischer selber einmal Anhängerin des Kommunismus waren, gewissermaßen einen intellektuellen Diskussions- und Klärungsprozess über die Geschichte des Sowjetkommunismus in Gang brachten. So wurde unter anderem immer wieder der Versuch unternommen, den »Großen Terror « in den 1930er-Jahren und hierbei die Rolle Stalins zu interpretieren. Das als "permanenter Terror« (Borkenau) bezeichnete Phänomen kostete Millionen von Menschen das Leben und zeigte seine schreckliche Seite beispielsweise in der Hungerskatastrophe in der Ukraine, dem Terror gegen die eigene Bevölkerung, der Parteisäuberung und den Moskauer Schauprozessen. Genauso war die Entwicklung des Stalinismus nach dem Ende des Zweiten Weltkrieges von zentraler Bedeutung. So wurden in diesem Zusammenhang sowohl der wieder in Gang gekommene Terror, die Säuberung der kommunistischen Parteien vor allem in den osteuropäischen Staaten als auch die Versuche Jugoslawiens unter Tito, einen von Moskau unabhängigen staatlichen Weg zu gehen, weit und breit thematisiert. Obwohl die Auseinandersetzung mit dem sowjetkommunistischen Herrschaftssystems im Monat unter der Ägide Laskys durchgehend von Bedeutung war, erreichte sie gleichwohl einen zwischenzeitlichen Höhepunkt nach dem Tod Stalins im März 1953. Auch in diesem Zusammenhang diente die Zeitschrift als ein Diskussionsforum für die internationalen Kommunismusexperten. So wichtig die Dokumentation und die Thematisierung zeitgenössischer Ereignisse in den osteuropäischen Ländern auch war - wie der Arbeiteraufstand vom 17. Juni 1953 in der »Ostzone« sowie die Unruhen in Polen und die Ungarische Revolution von 1956 -, kam hier nicht zuletzt ein intellektuelles, politisches und wissenschaftliches Gespräch in Gang, das sich in erster Linie mit der Frage beschäftigte, welche grundsätzlichen politischen Folgen der Tod Stalins für das sowjetkommunistische Herrschaftssystems hatte und wie das Wesen des sogenannten Nachstalinismus zu charakterisieren sei. Ebendiese Frage drängte sich neben den Ereignissen in den Satellitenstaaten vor allem aufgrund der Entwicklungen in der Sowjetunion selber auf. Denn die nach dem Tod Stalins einsetzenden Machtkämpfe um die Nachfolge des Partei- und Staatsoberhauptes, an dessen Ende Nikita Chruschtschow als Sieger hervorging, der Abbau des Terrors und der berühmte XX. Parteitag der KPdSU im Februar 1956, auf dem Chruschtschow offiziell die unter Stalin begangenen Verbrechen verurteilte, führten zu einer intensiven Dis-

nismus, in: Der Monat 2 (1950), H. 19, S. 76-79 (Rezension), sowie Raymond Aron, Der falsche Messias. Ein Schlußwort zu »The God that failed«, in: Der Monat 3 (1950), H. 26, S. 175-184. Des Weiteren siehe François Bondy, Der expropriierte Marxismus, in: Der Monat 2 (1950), H. 21, S. 309-313 (Rezension), sowie die drei Beiträge von Czeslaw Milosz: Murti-Bing, in: Der Monat 3 (1951), H. 35, S. 451-464, Die Baltischen Völker, in: Der Monat 4 (1951), H. 41, S. 451-466, und Ketman oder die Kunst des inneren Vorbehalts, in: Der Monat 5 (1953), H. 57, S. 227-242 (»Teil-Vorabdruck« von Verführtes Denken, Köln/ Berlin 1953); vgl. hierzu auch die entsprechenden Ausführungen von François Bondy, Rebellen als Musterschüler. Zu zwei neuen Essaybänden, in: Der Monat 5 (1953), H. 58, S. 407-411 (Rezension). 
kussion über das »Jenseits des Stalinismus« (Löwenthal), die politischen Implikationen und das Wesen des Regimes. ${ }^{277}$

Gleichwohl galt das Interesse im Monat nicht nur dem zeitgenössischen sowjetkommunistischen Herrschaftssystem einschließlich der osteuropäischen Satellitenstaaten. Genauso war die Redaktion darum bemüht, sich mit der Entwicklung der kommunistischen Parteien und Bewegung in Westeuropa auseinanderzusetzen, wobei in diesem Zusammenhang insbesondere die politische Situation in Italien ${ }^{278}$ und Frankreich ${ }^{279}$ ins Licht gerückt wurde.

Zweifelsohne besaß die Auseinandersetzung mit dem Phänomen des Kommunismus im Monat eine herausragende Bedeutung. Obwohl vor allem die Beschreibung und Analyse des Stalinismus und Nachstalinismus auf einem hohen Niveau erfolgte, bestand gleichwohl die Gefahr, dass die Zeitschrift ihre antikommunistische Funktion zu sehr in den Vordergrund rückt. Insofern war es der Redaktion um Lasky und Jaesrich frühzeitig darum zu tun, den prowestlichen und speziell den proamerikanischen Charakter der Zeitschrift zu unterstreichen. Vor diesem Hintergrund bemühte sich der Monat, die Vereinigten Staaten als Prototyp des Modells der westlichen Zivilisation darzustellen, ohne dass dies mit der gleichzeitig anvisierten europäischen Perspektive im Widerspruch stand. Ganz im Gegenteil, denn in der liberal-demokratischen Perspektive gehörten proamerikanisches und proeuropäisches Denken notwendig zusammen. Das proamerikanische Motiv stand selbstverständlich im $\mathrm{Zu}$ sammenhang mit der »Reorientation«-Funktion des Monat. Allerdings ging es hierbei nicht darum, ein eindimensionales und unkritisches Bild der Vereinigten Staaten zu zeichnen. So wurden vor allem die beiden hochbrisanten Probleme der amerikanischen Innenpolitik der 1950er-Jahre zur Sprache gebracht, nämlich das Phänomen des »Kommunistenjägers « Joseph McCarthy ${ }^{280}$ und die Benachteiligung der schwarzen Bevölkerungsgruppe, sprich: die »Negerfrage ${ }^{281}$

Im Hinblick auf das proamerikanische Denken standen Reflexionen über die Bedeutung der individuellen Freiheitsrechte als herausragenden Topos des liberal-demokratischen Systems im Zentrum. ${ }^{282}$ Das Freiheitsthema konnte wiederum umstandslos mit dem ausdrücklich hervorgehobenen und intensiv behandelten Aspekt von der Funktion der kulturellen Freiheit für den liberalen Westen insgesamt verbun-

277 Siehe hierzu die entsprechenden Ausführungen in Kap. IV.2.

278 Siehe exemplarisch Bernard Wall, Brief aus Rom: Ketzerei auf der Linken. Ein Bericht über die Krise in der KP Italiens, in: Der Monat 3 (1951), H. 31, S. 35-39.

279 Siehe exemplarisch Herbert Lüthy, Brief aus Paris: »Das Leben beginnt morgen«. Politische Gedanken zu einem unpolitischen Film, in: Der Monat 3 (1950), H. 26, S. 151-156.

280 Siehe Leslie A. Fiedler, Brief aus Amerika: Der streitbare Senator. Glanz und Elend Joe McCarthys, in: Der Monat 7 (1954), H. 74, S. 129-142, sowie Richard H. Rovere, Die letzten Tage Joe McCarthys. Abgesang auf einen Demagogen, in: Der Monat 11 (1953), H. 123, S. 39-50.

281 Siehe Nathan Glick, Die Leinwand predigt Toleranz. Rassenprobleme im amerikanischen Film, in: Der Monat 2 (1950, H. 21, S. 299-304; Walter Lippmann, Ein Brief William Faulkners, in: Der Monat 8 (1956), H. 91, S. 85 f.; Patrick O'Donovan, Der Prozeß des Martin Luther King. Brief aus Alabama, in: Der Monat 8 (1956), H. 92, S. 13-15; Alistair Cooke, Brief aus Amerika: Die Prüfung des Südens. Weiß und Schwarz in USA (Teil I), in: Der Monat 8 (1956), H. 96, S. 13-21, sowie Teil II in: Der Monat 8 (1956), H. 97, S. 19-22; Rovere, Die Neun von Little Rock, in: Der Monat 8 (1957), H. 109, S. 78 f.

282 Hochgeschwender, Freiheit in der Offensive?, S. 179. 
den werden und spielte nicht zuletzt auf dem Berliner »Kongreß für kulturelle Freiheit« im Juni 1950 eine herausragende Rolle. ${ }^{283}$

Individuelle und kulturelle Freiheit waren im Monat Grundkonstituenten nicht nur des amerikanischen politischen Systems, sondern von Westlichkeit überhaupt. Gleichzeitig bot es den adäquaten Rahmen, um in dem Organ verschiedene sozioökonomische Ordnungsmodelle zu beleuchten. Gesamtgesellschaftliche Planbarkeit, insbesondere im wirtschaftlichen Bereich, wurde hierbei keineswegs radikal infrage gestellt, insofern es sich innerhalb des von der Freiheitsthematik vorgegebenen liberalen Bezugssystems bewegte. In diesem Zusammenhang wurde beispielsweise die Frage eines >demokratischen Sozialismus als antithetisches Modell zum stotalitären Kommunismus $<$ thematisiert ${ }^{284}$ als auch die Frage, inwiefern in der >sozialen Marktwirtschaft noch die »alten« Gegensätze zwischen Kapitalismus und Sozialismus bestanden. ${ }^{285}$

Die zahlreichen Beiträge im Monat, die sich mit dem politischen, kulturellen und intellektuellen Leben in den Vereinigten Staaten beschäftigten, sollten dazu beitragen, den in Gang gebrachten Demokratisierungsprozess in Westdeutschland zu fördern. Vor dem Hintergrund des anvisierten Zieles, mit der Zeitschrift die deutschen Intellektuellen und das Bildungsbürgertum für die westlich-pluralistische Form der Demokratie zu gewinnen, galt es in diesem Kontext vor allem, nicht nur den weit verbreiteten »Antiamerikanismus « zu bekämpfen, sondern ausdrücklich dem in diesen Kreisen grassierenden Vorurteil einer kulturlosen Nation entgegenzutreten. ${ }^{286}$ Folglich sah es die Redaktion als eine zentrale Aufgabe an - so wie es die von der amerikanischen Besatzungsmacht herausgegebene Neue Zeitung sozusagen vormachte -, die deutschen Leser insbesondere mit den renommiertesten amerikanischen Schriftstellern aus der ersten Hälfte des 20 . Jahrhunderts ${ }^{287}$ und den »großen « Werken der zeitgenössischen

283 Siehe hierzu das Kap. I.5.

284 Siehe vor allem die Diskussion »Der Streit um den Sozialismus. Sechs Stellungnahmen«, in: Der Monat 1 (1949), H. 5, S. 3-33 (mit Beiträgen von Wilhelm Röpke, Ernst Tillich, Sidney Hook, Friedrich A. Hayek, Joseph A. Schumpeter und Willy Brandt); vgl. auch Ignazio Silone, Der Sozialismus am Kreuzweg. Ein politisches Bekenntnis, in: Ebd., H. 10, S. 84-89.

285 Siehe C. A. R. Crosland, Ist das noch Kapitalismus? Ein englischer Sozialist über das Großbritannien von heute, in: Der Monat 9 (1957), H. 103, S. 9-18, sowie Gustav Klingelhöfer, Kapitalismus ohne Schrecken ... und der Sozialismus von heute, in: Ebd. 10 (1958), H. 116, S. 8-18.

286 Siehe vor allem Robert Pick, Mit europäischen Augen. Amerika im Spiegel der europäischen Literaturkritik, in: Der Monat 2 (1950), H. 18, S. 658-664; Irving Howe, Der Nachkriegsroman in Amerika. Eine Bestandsaufnahme um die Jahrhundertmitte, in: Der Monat 3 (1951), H. 34, S. 424-428; Leslie A. Fiedler, Der »gute Amerikaner«. Ein Amerikaner unter europäischen Intellektuellen, in: Der Monat 6 (1954), H. 69, S. 287-289; Walter Hasenclever, Zornig - aber nicht jung. Amerikas »Beat Ceneration«, in: Der Monat 11 (1958), H. 121, S. 74-78.

287 Siehe exemplarisch Thomas Wolfe (1900-1938), in: Der Monat 1 (1948), H. 1, S. 56-77 (Auszüge aus dem Werk), und hierzu die beiden literarischen Porträts: H. M. Ledig-Rowohlt, Thomas Wolfe in Berlin, in: Der Monat 1 (1948) H. 1, S. 69-77, sowie Alfred Kazin, Rhetorik und Verzweiflung, in: Der Monat 1 (1948), H. 1, S. 78-80; Granville Hicks, Der Romancier und die Macht. Über John Dos Passos, in: Der Monat 4 (1952), H. 40, S. 414-420. 
amerikanischen Literatur vertraut $\mathrm{zu}$ machen, ${ }^{288}$ ohne das amerikanische Theater ${ }^{289}$ oder den amerikanischen Film ${ }^{290}$ zu vernachlässigen.

Das proamerikanische Motiv im Monat hatte, wie gesagt, seine Bedeutung nicht zuletzt in der "Re-orientation «-Funktion der Zeitschrift. Dieser zentralen Aufgabe diente zudem die Thematisierung der amerikanischen Philosophie, die im Grunde genommen zu den ideologischen Grundlagen der Zeitschrift gehörte und eine wichtige Bedeutung in der Auseinandersetzung mit der deutschen Vergangenheit besaß. Denn damit die Zeitschrift einen Beitrag zur »Verwestlichung« Deutschlands leisten konnte, war die Auseinandersetzung mit der Geschichte des >Dritten Reiches` unabdingbar, wollte man die antitotalitäre Position nicht nur auf den zeitgenössischen Sowjetkommunismus beziehen. Hier bot sich vor allem die Thematisierung des Konzeptes der pragmatisch ausgerichteten Philosophie von John Dewey an, mit der auf einer geistesgeschichtlichen Ebene der Nationalsozialismus aus einer genuin antidemokratischen Tradition interpretiert werden konnte. Deweys Philosophie, die als der klarste Ausdruck des amerikanischen Liberalismus galt, ${ }^{291}$ wurde explizit und implizit der deutschen Philosophie entgegengestellt und die in eine Kritik der »totalisierenden Staats- und Ganzheitsfixierung der hegelianischen Ontologie, Erkenntnislehre und Staatsphilosophie« (Hochgeschwender) mündete. Neben Fichte und Hegel zählte für den Monat auch die Philosophie Nietzsches zu den geistigen Wegbereitern des Nationalsozialismus, ${ }^{292}$ in dessen Folge nicht nur der Nihilismus Nietzsches, ${ }^{293}$ sondern auch der Existenzialismus Martin Heideggers ${ }^{294}$ und das Denken von Ernst Jünger ${ }^{295}$ thematisiert wurden.

Gleichwohl blieb es im Monat im Hinblick auf die nationalsozialistische Vergangenheit keinesfalls bei der Auseinandersetzung mit der deutschen Philosophie bzw. einflusseichen philosophischen und politischen Denkern. Einerseits ging das Organ

288 Siehe exemplarisch den dreiteiligen Abdruck der Erzählung William Faulkners Der Bär im Monat (3 [1951], H. 29, S. 510-528 [I], H. 30, S. 603-631 [II], sowie H. 31, S. 58-84 [III]).

289 Siehe bes. Hans Sahl, Kinder und Heilige. Ein New Yorker Theaterbericht, in: Der Monat 2 (1950), H. 18, S. 647-650, sowie Werner Oehlschläger, Saison am Broadway. Bemerkungen zum amerikanischen Theaterleben, in: Ebd. 3 (1951), H. 29, S. 533-541.

290 Siehe bes. Georg Gerster, Hollywood als Seelenzustand. Anmerkungen zu zwei Studien über den amerikanischen Film, in: Der Monat 4 (1952), H. 40, S. 408-413; vgl. auch Friedrich Luft, Vom großen schönen Schweigen. Arbeit und Leben des Charles Spencer Chaplin, in: Der Monat 4 (1952), H. 44, S. 184-192.

291 Vgl. Sidney Hook, John Dewey. Ein Porträt des Altmeisters des amerikanischen Geisteslebens, in: Der Monat 1 (1949), H. 6, S. 40-46; Alfred North Whitehead, John Dewey zum 90. Ceburtstag, in: Der Monat 2 (1949), H. 13, S. 23 f.; John Dewey, Vom Ursprung des Philosophierens. Philosophie im Wandel der Auffassungen, in: Der Monat 2 (1949), H. 13, S. 25-35; Joachim G. Leithäuser, Erziehung zur Zukunft, in: Der Monat 2 (1950), H. 20, S. 218-220 (Rezension); Ludwig Marcuse, Eine Theorie der Praxis. Amerikanischer und deutscher Pragmatismus, in: Der Monat 8 (1956), H. 88, S. 33-45.

292 Vgl. Marianne Regensburger, Hegel und der europäische Nihilismus, in: Der Monat 3 (1951), H. 32 , S. 202-205 (Rezension).

293 Vgl. Albert Camus, Nietzsche und der Nihilismus, in: Der Monat 4 (1951), H. 39, S. 227-236.

294 Vgl. Kurt Rossmann, Martin Heideggers Holzwege, in: Der Monat 2 (1950), H. 21, S. 236-245.

295 Vgl. bes. Peter de Mendelssohn, Gegenstrahlungen. Ein Tagebuch zu Ernst Jüngers Tagebuch, in: Der Monat 2 (1949), H. 14, S. 149-174, sowie Alfred Weber, Flucht in die Wildnis. Randbemerkungen zu Ernst ]üngers politischen Schriften, in: Der Monat 3 (1951), H. 29, S. 542-545. 
in zahlreichen Beiträgen auf die Geschichte des >Dritten Reiches ein, andererseits geriet auch der facettenreiche Umgang mit der nationalsozialistischen Vergangenheit in der Bundesrepublik in den Fokus des Interesses, ${ }^{296}$ der nicht zuletzt dem Ziel diente, das demokratische System zu stabilisieren und einen Beitrag zur Konstitution einer demokratischen, politischen Kultur im Nachkriegsdeutschland zu leisten. Um nur einige Beispiele zu nennen. Vor dem Hintergrund der grundsätzlichen Erinnerung an die Opfer der »Hitler-Diktatur« im Allgemeinen und die nationalsozialistischen Judenverfolgungen und den sogenannten deutschen Verbrechen an den deutschen und europäischen Juden im Besonderen - wie es beispielsweise der erste Bundespräsident Theodor Heuss beim feierlichen Staatsakt zur Einweihung eines Mahnmales für die Opfer des Konzentrationslagers Bergen-Belsen am 30. November 1952 zum Ausdruck brachte ${ }^{297}$ - ging es der Zeitschriftenredaktion um Lasky durchaus darum, Versuche von Exponenten der »Neuen Rechten«, ebendiese Massenverbrechen, kurz: Auschwitz, zu verharmlosen, indem man sie umstandslos mit anderen Verbrechen auf »eine Stufe« (Alfred Kellner) stellte (wie zum Beispiel der Vertreibung der Deutschen aus Polen und dem Sudetenland oder den Verbrechen des Stalinismus), vehement zu kritisieren. ${ }^{298}$

Der millionenfache Mord an den europäischen Juden war im Monat auch Anlass, sich mit dem zukünftigen Verhältnis der Deutschen zu den Juden in Deutschland sowie zum Staat Israel zu beschäftigen. Hierbei sprach man sich sowohl für eine Aussöhnung zwischen »uden und Deutschen«als auch für eine »Wiedergutmachungspolitik« aus, nicht zuletzt gegenüber dem Staat Israel. ${ }^{299}$ In diesem Zusammenhang wurde in einigen wenigen Beiträgen auch die »deutsche Schuldfrage« auf einer primär abstrakten Ebene angesprochen, wobei man sich ausdrücklich gegen eine Kollektivschuld des deutschen Volkes wandte. ${ }^{300}$ Angesichts der nationalsozialistischen Verbrechen an den Juden sprach die »Lasky-Zeitschrift« das in der bundesrepublikanischen Bevölkerung weithin verbreitete Phänomen, Auschwitz zu verdrängen, zumindest an. ${ }^{301}$ Der Monat setzte sich auch mit dem postnationalsozialistischen Antisemitismus bzw. dem Phänomen des Antisemitismus in der Bundesrepublik auseinander, wie zum Beispiel aus Anlass der Schändung der Kölner Synagoge am Weihnachtsabend $1959^{302}$ oder anhand

296 Vgl. unter diesem Aspekt exemplarisch das Symposium »Erwacht Deutschland schon wieder? Der neue deutsche Nationalismus und seine Gefahren, in: Der Monat 1 (1949), H. 8/9, S. 3-64 (mit Beiträgen von Walter L. Dorn, Richard H. S. Crossmann, Raymond Aron, Dolf Sternberger, Peter de Mendelssohn, Wilhelm Röpke, Eugen Kogon, Franz Borkenau u. a.) sowie Norbert Muhlen (schreibt aus Hamburg): Anne Franks Heimkehr, in: Der Monat 9 (1957), H. 103, S. 79-82.

297 Theodor Heuss, Ein Mahnmal, in: Der Monat 5 (1953), H. 52, S. 355-358.

298 Vgl. beispielsweise a[lfred] k[ellner], Gefährliche Gleichsetzungen, in: Der Monat 3 (1951), H. 30, S. $660 \mathrm{f}$.

299 Siehe bes. Elliot Cohen, Deutsche und Juden. Eine Rede in Berlin, in: Der Monat 3 (1951), H. 28, S. 375379. Vgl. auch Erich Lüth, Brief aus Loccum: Deutsche und Juden heute. Fazit einer Tagung, in: Der Monat 10 (1957), H. 110, S. 46-50, und Hellmut Gollwitzer, Zehn Jahre Israel. Deutsche und Juden heute, in: Der Monat 10 (1958), H. 119, S. 52-61.

300 Vgl. exemplarisch Rudolf Hagelstange, Sühne und Ehre, in: Der Monat 4 (1952), H. 42, S. 584-589.

301 So zum Beispiel Arthur Koestler in seinem Beitrag Politische Neurosen in: Der Monat 6 (1953), H. 63 , S. $228 \mathrm{f}$.

302 Vgl. Klaus Harpprecht, Im Keller der Gefühle. Gibt es noch einen deutschen Antisemitismus, in: Der Monat 11 (1959), H. 128, S. 13-20. 
des »Falles Harlan«, bei dem es Ende der 1940er-/Anfang der 1950er-Jahre zu zahlreichen Protestaktionen gegen den Regisseur des berüchtigten antisemitischen Hetzund Propagandafilms JuD Süss aus den Jahren des >Dritten Reiches`, Veit Harlan, kam, die insbesondere von einigen Prominenten, Teilen der SPD, den Gewerkschaften, den Studentenverbänden und der Gesellschaft für Christlich-Jüdische Zusammenarbeit getragen wurden. Harlan avancierte seinerzeit sowohl zu einem Symbol des in der frühen Bundesrepublik grassierenden Antisemitismus als auch für den sich wieder um sich greifenden Nazismus. ${ }^{303}$

Der »Fall Harlan« war auch ein herausragendes Beispiel dafür, dass sich Der Monat mit bestimmten politischen Skandalen auseinandersetzte, die die nationalsozialistische Vergangenheit betrafen und grundsätzlich von Bedeutung waren für die Konstitution einer demokratisch-politischen Kultur in der Bundesrepublik. Als zweites Beispiel sei an dieser Stelle der »Remer-Prozess« genannt, bei dem es Anfang 1950 auch und vor allem um die Frage der Rehabilitierung des politisch-militärischen Widerstandes gegen die NS-Diktatur ging und der seinerzeit in der bundesrepublikanischen Öffentlichkeit große Aufmerksamkeit fand. Die Thematisierung der näheren Umstände und des Verlaufs dieses gerichtlichen Verfahrens, bei dem das Landgericht Braunschweig den ehemaligen Generalmajor und jetzigen zweiten Vorsitzenden der Sozialistischen Reichspartei (SRP), Otto Ernst Remer, wegen übler Nachrede zu einer dreimonatigen Gefängnisstrafe verurteilte, weil er die Widerstandskämpfer des 20. Juli als »Landesverräter « beschimpft hatte, ${ }^{304}$ hatte für den Monat einen entscheidenden Grund: Hier ging es um die ausdrückliche Rehabilitierung des »20. Juli« und die Legitimierung des Widerstandes gegen das »Hitler-Regime«. Aus diesem Grund widmete sich die Zeitschrift auch intensiv ebendiesem Thema. ${ }^{305}$ Das Ziel bestand darin, den Lesern die Männer und Frauen der Widerstandsgruppen um den Oberst Claus Graf Schenk von Stauffenberg als Repräsentanten eines »anderen Deutschlands« hervorzuheben, das für die Konstitution einer politischen Kultur und die Errichtung einer freiheitlichen Demokratie in der Bundesrepublik eine wichtige Funktion besaß.

Insbesondere in Anbetracht dieser Tatsache stand der Monat zweifelsohne in der Tradition mit den oben exemplarisch genannten politisch-kulturellen Zeitschriften Die Wandlung, Der Ruf und den Frankfurter Heften. Allerdings existierte in diesem Kontext ein gravierender Unterschied. Brachten die drei Zeitschriften der »Stunde Null« eine zum Teil radikale Skepsis und Kritik gegenüber den Nürnberger Prozessen und den Entnazifizierungsmaßnahmen zum Ausdruck, die sich in erster Linie auf die amerikanische Besatzungspolitik bezog und alsbald von weiten Teilen der deutschen Bevölkerung mitgetragen wurde, verzichtete der Monat darauf, diesen Themenkomplex genauer in den Blickpunkt zu rücken. So spielte für die Redaktion um Lasky die ausdrückliche Beschäftigung mit den Nürnberger Prozessen ebenso keine Rolle, ob-

303 Siehe Hans Schwab-Felisch, Die Affäre Harlan, in: Der Monat 3 (1951), H. 28, S. 414-422, sowie Clara Menck, Brief aus Freiburg: Studenten gegen Harlan, in: Der Monat 4 (1952), H. 42, S. 573-583; vgl. auch die Leserbriefe unter der Überschrift Veit Harlans Unmenschlichkeit in: Der Monat 3 (1951), H. 29, S. 555-558.

304 Siehe hierzu Rüdiger Proske, Brief aus Braunschweig: Prozeß um den 20. Juli. Die Braunschweiger Verhandlungen gegen Otto Ernst Remer, in: Der Monat 4 (1952), H. 43, S. 16-21.

305 Siehe hierzu in Kap. IV.1 vor allem den Abschnitt 1.7, in dem zahlreiche in diesem Zusammenhang veröffentlichte Beiträge aufgeführt werden. 
wohl beispielsweise der »Wilhelmstraßen-Prozess « erst im Laufe des Jahres 1949 abgeschlossen wurde, wie auch die tatsächliche amerikanische Besatzungspolitik keine nennenswerte Bedeutung besaß. Eine Ausnahme stellte hier ein Beitrag dar, der eine radikale Kritik an einem der auflagenstärksten Bücher der unmittelbaren Nachkriegsjahre enthielt, das nicht zuletzt deshalb zu einem »Beststeller« werden konnte, weil es sich nämlich um eine polemische Abrechnung mit der tatsächlichen amerikanischen Besatzungspolitik im Allgemeinen und den Entnazifizierungsmaßnahmen der USMilitärregierung im Besonderen handelte. Die Rede ist von dem 1951 erschienenen Buch Der Fragebogen von Ernst von Salomon. ${ }^{306}$

Wie zum Ausdruck gebracht werden sollte, war der Monat eine genuine Zeitschrift des Kalten Krieges. In Anbetracht der Tatsache, dass sich die bis dahin gültige Reeducation-Politik in den Augen der amerikanischen Besatzungsbehörde als nicht mehr zeitgemäß erwies, besaß das Organ im Hinblick auf die Deutschlandpolitik einerseits die Aufgabe, das fortan maßgebliche »Reorientations-Projekt« in einem ganz speziellen Sinne zu unterstützen. Hierbei ging es dem Monat in der frühen Bundesrepublik insbesondere darum, deutsche Intellektuelle und das intellektuelle Bildungsbürgertum beziehungsweise eine neue Generation deutscher Intellektueller für die westlich-pluralistische Form der Demokratie zu gewinnen, wobei die Freiheitsrechte amerikanischer Provenienz im Mittelpunkt standen. Andererseits hatte der Monat von Anbeginn eine bestimmte Funktion in der Konfrontation des westlichen Systems mit der unbestrittenen Führungsmacht der Vereinigten Staaten auf der einen Seite und dem sowjetkommunistischen System einschließlich seiner Satellitenstaaten auf der anderen Seite. Hierbei sollte die antikommunistische Propaganda auf einem hohen intellektuellen Niveau geführt werden. Letzten Endes ergänzten sich die beiden Funktionen der »Reorientation « und des Antikommunismus auf eine ganz spezifische Weise, und das wirkte sich selbstverständlich auf die inhaltliche Ausrichtung aus. Vor dem Hintergrund der grundsätzlichen antitotalitären Position der Zeitschrift folgte daraus zudem sowohl eine Auseinandersetzung mit dem Nationalsozialismus und dem Sowjetkommunismus als auch mit der Totalitarismustheorie.

Dies wurde von dem Tübinger Historiker Michael Hochgeschwender auf überzeugende Weise in seiner Studie Freiheit in der Offensive? Der Kongreß für kulturelle Freiheit und die Deutschen zur Sprache gebracht und hervorgehoben. Was hier allerdings nicht ausdrücklich untersucht wurde, war der Fragenkomplex, wie die beiden totalitären Herrschaftssysteme des Nationalsozialismus und des Sowjetkommunismus tatsächlich beschrieben und analysiert wurden, d. h. vor allem, welches Bild von den beiden Herrschaftsregimen gezeichnet wurde, wie der konkrete Vergleich der beiden Regime letzten Endes ausfiel und welche Rolle die zeitgenössischen Totalitarismustheorien in diesem Zusammenhang spielten. Obwohl das facettenreiche Totalitarismusphänomen rechter und linker Provenienz im Monat in den Fokus des Interesses rückte - worauf Hochgeschwender zu Recht hinwies -, ist bisher auch eine nähere Untersuchung ausgeblieben, welche Autoren zu ebenjenem Themenkomplex im Monat veröffentlichten und - allgemein gesprochen - ob die »Lasky-Zeitschrift« die wissenschaftlichen und historisch-politischen Erkenntnisse seiner Mitarbeiter bei der (vergleichenden) 
Analyse des nationalsozialistischen und sowjetkommunistischen Herrschaftssystems nutzte und auf die zeitgenössischen Forschungsergebnisse zurückgriff.

\subsection{Die Rolle der Totalitarismustheorie in der frühen Bundesrepublik}

Nach dem Ausbruch des Ost-West-Konfliktes erwies sich die klassische Totalitarismustheorie (Arendt; Friedrich/Brzezinski) in den 1950er-Jahren als geeignetes Instrument, um die Westdeutschen im Kampf gegen den »neuen« totalitären Feind aus der Sowjetunion an die Seite der westlichen Staaten zu stellen sowie ein eindimensionales und personenzentriertes Bild des nationalsozialistischen Herrschaftssystems zu zeichnen, in dessen Zentrum Hitler stand. Anders gesagt: Die klassische Totalitarismustheorie besaß im Kalten Krieg in der Bundesrepublik insbesondere eine antikommunistische Funktion, die ein Grund dafür war, dass die Analyse des »Führerstaates« einen reduktionistischen Charakter aufwies.

Dabei konfrontierten bereits die 13 Nürnberger Prozesse die westdeutsche Nachkriegsgesellschaft nicht nur mit den nationalsozialistischen Massenverbrechen, sondern zeigten auch, dass das Unrechtsregime nicht auf eine kleine kriminelle Führungsclique um Hitler sowie die Herrschaftsinstitutionen der SS und Gestapo reduziert werden konnte. Gleichwohl beharrten die Vertreter der "Outlaw-Theorie« auf jener Vorstellung, in der der deutschen Bevölkerung der Platz eines »verführten Volkes« zugewiesen wurde, das für den begangenen Völkermord an den europäischen Juden und für die Kriegsverbrechen nicht zur Verantwortung gezogen werden dürfe. Zudem vertraten die deutschen Historiker und Publizisten die Auffassung, dass die »Hitler-Diktatur« einen Einbruch sowohl in der deutschen als auch der europäischen Geschichte darstellte, und betrachteten ihn als einen >Betriebsunfalk der Weltgeschichte, der sich zwar in Deutschland ereignete, allerdings in jedem Land möglich gewesen wäre. So stellten auch und vor allem Friedrich Meineckes einflussreicher Essay Die deutsche Katastrophe ${ }^{307}$ aus dem Jahre 1946 und - in stärkerem Maße - das zwei Jahre später erschienene Buch Europa und die deutsche Frage von Gerhard Ritter ${ }^{308}$ den Versuch dar, den Nationalsozialismus aus einer negativen gesamteuropäischen Entwicklung heraus zu erklären, die mit der Französischen Revolution ihren Anfang nahm und die parallel $\mathrm{zu}$ der grundsätzlich sgesunden existiert hätte. Das >Dritte Reich erschien als eine Katastrophe, die über Deutschland hereingebrochen war, und als ein historischer Zufall, der sich größtenteils auf den »Dämon« Hitler zurückführen ließ. Zudem setzten sich führende Historiker in Westdeutschland auch weniger mit der konkreten Geschichte des Nationalsozialismus auseinander als vielmehr mit dem Widerstand gegen das »Hitler-System«. In der bürgerlich-konservativen Opposition aus Offizieren, Kirchenvertretern und Beamten erkannten sie quasi ein »anderes Deutschland« und meinten insofern einen Ausweg aus der NS-Geschichte gefunden zu haben, als hiermit ein Anknüpfungspunkt für den

307 Friedrich Meinecke, Die deutsche Katastrophe, Wiesbaden 1946.

308 Gerhard Ritter, Europa und die deutsche Frage. Betrachtungen über die geschichtliche Eigenart des deutschen Staatsdenkens, München 1948. 